\title{
Some Groups Generated by Transvections
}

\author{
By \\ JaCk McLaughitin*)
}

Let $V$ be a vector space of dimension $n \geqq 2$ over a field $K$. For each pair of subspaces $P \subseteq H$ of dimension 1 and $n-1$ respectively the subgroup of $S L(V)$ generated by those transvections $\tau$ with $H=\operatorname{Ker}(\tau-1), P=\operatorname{Im}(\tau-1)$ will be denoted by $X(P, H)$. Drawing from the language of Lie theory, John Thompson has christened these subgroups of root type, and he has asked which subgroups of $S L(V)$ having only 1 as a normal unipotent subgroup are generated by subgroups of root type. In this note we give the following partial answer.

Theorem. Suppose $K \neq \boldsymbol{F}_{2}, \operatorname{dim} V \geqq 2$, and that $G$ is a subgroup of $S L(V)$ which is generated by subgroups of root type. Suppose also that 1 is the only normal unipotent subgroup of $G$. Then for some $s \geqq 1, V=V_{0} \oplus V_{1} \oplus \cdots \oplus V_{s}, G=G_{1} \times \cdots \times G_{s}$, and

(a) The $V_{i}$ are stable for the $G_{j}$.

(b) $G_{i} \mid V_{j}=1$ if $i \neq j$.

(c) $G_{i} \mid V_{i}=S L\left(V_{i}\right)$ or $S p\left(V_{i}\right)$.

For $K=F_{2}$ there are many other examples, but we have been unable to construct a definitive list.

The proof of the theorem will be made by examining the action of $G$ on the set of subspaces of $V$. We begin by describing the terminology we shall use. For the time being $K$ is any field and we suppose only that $\operatorname{dim} V \geqq 2$ and $G \subseteq S L(V)$ is generated by subgroups of root type. (We take this to mean $G \neq 1-G$ contains subgroups of root type.) This hypothesis will always be in force. Others will be explicitly stated when used. We say $P$ is a center (for $G$ ) if, for some $H, X(P, H) \subseteq G$; likewise $H$ is an axis (for $G$ ). We will also say $H$ is an axis for $P$ and $P$ is a center for $H$. For each center $P, a(P)$ is the intersection of the axes for $P$; dually $c(H)$ is the sum of the centers for $H$. $C$ is the set of centers for $G$ and $\mathfrak{A}$ is the set of axes for $G$. If $U$ is a subspace of $V, G_{U}$ is the stabilizer of $U$ in the action of $G$ on the subspaces of $V$. Finally, since we are primarily concerned with subspaces rather than vectors and point is easier to say than one-dimensional subspace, we shall use the term point for one-dimensional subspace.

Lemma 1. If $\operatorname{dim} V=2$ and $|\subseteq|>1$ then $G=S L(V)$.

*) Work supported in part by a grant from the National Science Foundation. 


\section{Proof. Clear.}

Lemma 2. Let $P$ and $Q$ be centers with $\operatorname{dim} a(P) \leqq \operatorname{dim} a(Q)$ and $P \nsubseteq a(Q)$. Then $Q \nsubseteq a(P)$ and if $U \supseteqq P+Q$ then $G_{U, P+Q} \mid P+Q$ contains $S L(P+Q)$. In particular every point on $P+Q$ is a center.

Proof. If $Q \subseteq a(P)$, then $Q$ and so $a(Q)$ is fixed by all $X(P, H) \subseteq G$. Since $P \nsubseteq a(Q)$, $a(Q)$ is on all axes of $P-a(Q) \subseteq a(P)$. Then $a(Q)=a(P)$ against $P \nsubseteq a(Q)$. Thus $G$ contains subgroups $X(P, H), X(Q, K)$ with $P \nsubseteq K, Q \nsubseteq H$. If $U \supseteq P+Q$, each of these subgroups is in $G_{U, P+Q}$. The second conclusion then follows from Lemma 1.

We can now give the first approximation to the theorem.

Lemma 3. Suppose $\mathbb{C}$ consists of all points, $\mathfrak{A}$ consists of all hyperplanes, and $G$ is transitive on these two sets. Then either $G=S L(V)$ or $G=S p(V)$.

Proof. Suppose first that each $P$ has a unique axis $\delta P$ and each $H$ has a unique center $\delta H$. Then $a(P)=\delta P, c(H)=\delta H$. By Lemma 2, $P \subseteq \delta Q$ if and only if $Q \subseteq \delta P$. That is, $P \subseteq H$ if and only if $\delta H \subseteq \delta P$ and $\delta$ extends to a null polarity on the set of subspaces of $V$. If $X(P, \delta P) \subseteq G$ and $\sigma \in G$ then $\sigma X(P, \delta P) \sigma^{-1}=$ $=X(\sigma P, \sigma \delta P) \subseteq G$. Hence $\sigma \delta P=\delta \sigma P$ and $G$ is in the group of $\delta$. It is clear that $G$ contains all transvections commuting with $\delta$ and hence $G=S p(V)$.

Next suppose $a(P)$ is not a hyperplane. Let $H$ be an axis for $P$ and choose $Q \subseteq H$, $Q \nsubseteq a(P)$. Then by Lemma 2 , we have $\sigma \in G_{H}$ with $\sigma P=Q$. Hence every point of $H$ which is not on $a(P)$ is a center for $H$ and consequently $c(H)=H$. Then by duality $a(P)=P$ for all points $P$. Now the above shows that all points of $H$ are centers for $H$. Thus $G$ contains all transvections and $G=S L(V)$.

Finally if we start with the assumption that $c(H)$ is not a point and dualize the above we again get $G=S L(V)$.

Remark 1. If the field $K$ is finite one can draw the same conclusion from the (formally) weaker hypothesis that $Q$ is irreducible and each $P$ in $V$ is the center for some transvection in $G$.

Remark 2. A corollary of the lemma is that $S p(V)$ is a maximal subgroup of $S L(V)$ (except, of course, when $\operatorname{dim} V=2$ ).

Lemma 4. Suppose $G$ is transitive on $C$. If $P, S$ are distinct members of $\mathbb{S}$ with $S \subseteq a(P)$ there is a center $Q$ with $P \nsubseteq a(Q)$ and $S \nsubseteq a(Q)$.

Proof. If not then for all $T$ in the $G_{P}$-orbit of $S$ we have $P \nsubseteq a(Q)$ implies $T \subseteq a(Q)$. Choose $X(R, K) \subseteq G$. If $P \subseteq a(R)$ then $X(R, K) \subseteq G_{P}$ and $X(R, K)$ fixes the $G_{P}$-orbit of $S$. If $P \nsubseteq a(R)$ then $X(R, K)$ fixes each member of the $G_{P}$-orbit of $S$. Since $G$ is generated by the $X(R, K)$ we contradict the transitivity of $G$ on $\mathcal{C}$.

Lemma 5. Take $K \neq F_{2}$ and suppose $G$ is transitive on $\mathbb{C}$. If $P, S \in \mathbb{S}$ then each point on $P+S$ is in $C$.

Proof. Since $G$ is transitive on $(S$, all $a(P)$ have the same dimension and hence by Lemma $2, P \nsubseteq a(S)$ if and only if $S \nsubseteq a(P)$ and we have nothing to prove if $S \nsubseteq a(P)$. Suppose $S \neq P, S \subseteq a(P)$, and choose $Q \in C$ so $S \nsubseteq a(Q)$ and $S \nsubseteq a(P)$ (Lemma 4$)$. 
Set $U=P+Q+S . G$ contains the subgroups $X(P, H), X(S, J)$, and $X(Q, K)$ with $P \nsubseteq K, Q \nsubseteq H$, and $Q \nsubseteq J$. Since $P+S \subseteq a(P), P+S=a(P) \cap U=H \cap U$. Since $X(P, H)$ and $X(Q, K)$ fix $U, a(P) \cap U$ and $a(Q) \cap U$ have the same dimension and therefore $a(Q) \cap U=K \cap U$ and $S \nsubseteq K$. We want to determine the group induced on $U$ by $X(P, H), X(S, J)$ and $X(Q, K)$. Although the result is wellknown and can be easily obtained synthetically, we sketch the argument using matrices. Set $R=U \cap H \cap K$ and choose a base for $U$ consisting of $x_{1}, x_{2}, x_{3}$ from $R, P, Q$ respectively. The group induced on $U$ by $X(P, H)$ and $X(Q, K)$ will then be represented by the group of matrices of the form

where

$$
\left(\begin{array}{lll}
1 & 0 & 0 \\
0 & u_{1} & u_{2} \\
0 & v_{1} & v_{2}
\end{array}\right)
$$

$$
\left(\begin{array}{ll}
u_{1} & u_{2} \\
v_{1} & v_{2}
\end{array}\right)
$$

is an arbitrary member of $S L_{2}(K)$. The group induced by $X(S, J)$ will be represented by the matrices

$$
\left(\begin{array}{lll}
1 & 0 & t a \\
0 & 1 & t u \\
0 & 0 & 1
\end{array}\right)
$$

where $a u \neq 0$ and $t$ is arbitrary in $K$. Putting these two groups together we see that $G$ is transitive on the points of $U$ other than $R$ so that all such points are centers for $G$. We also see that if $L$ is a complement for $R$ in $H \cap K$ and $J \supseteq L$ then $R$ is also a center for $G$ and we are done. Thus we suppose $J \cap L$ is a hyperplane of $L$, choose a complement, $T=\left\langle x_{4}\right\rangle$, and with respect to the base $\left\{x_{1}, x_{2}, x_{3}, x_{4}\right\}$ for $U+T$ we again look at the matrices determined by our three groups - now acting on $U+T$. As before $X(P, H)$ and $X(Q, K)$ will yield the group of

$$
D(A)=\left(\begin{array}{ccc}
1 & 0 & 0 \\
0 & A & 0 \\
0 & 0 & 1
\end{array}\right)
$$

where $A$ is arbitrary in $S L_{2}(K)$, and from $X(S, J)$ we get a matrix

where

$$
M=\left(\begin{array}{lll}
1 & \alpha & c \\
0 & Z & \beta \\
0 & 0 & 1
\end{array}\right)
$$

and $a b c \neq 0$. Set

$$
\alpha=\left(\begin{array}{ll}
0 & a
\end{array}\right), \quad Z=\left(\begin{array}{ll}
1 & z \\
0 & 1
\end{array}\right), \quad \beta=\left(\begin{array}{l}
b \\
0
\end{array}\right)
$$

$$
N(A)=D(A) D\left(Z^{-1}\right) M D\left(A^{-1}\right)=\left(\begin{array}{ccc}
1 & \alpha A^{-1} & c \\
0 & 1 & A \beta \\
0 & 0 & 1
\end{array}\right) .
$$


Then for $A, B \in S L_{2}(K)$ we have

$$
\begin{aligned}
D\left(Z^{-1}\right) M N(A)(N(B))^{-1} & =\left(\begin{array}{ccc}
1 & \alpha\left(1+A^{-1}\right) & 2 c+\alpha A \beta \\
0 & 1 & (A+1) \beta \\
0 & 0 & 1
\end{array}\right)\left(\begin{array}{ccc}
1 & -\alpha B^{-1} & -c \\
0 & 1 & -B \beta \\
0 & 0 & 1
\end{array}\right)= \\
& =\left(\begin{array}{cccc}
1 & \alpha\left(1+A^{-1}-B^{-1}\right) & c+\alpha A \beta-\alpha\left(1+A^{-1}\right) B \beta \\
0 & 1 & (A+1-B) \beta \\
0 & 0 & 1
\end{array}\right) .
\end{aligned}
$$

Take

so

$$
A=\left(\begin{array}{cc}
-1 & 1 / x \\
-x & 0
\end{array}\right) \text { and } B=1+A=\left(\begin{array}{cc}
0 & 1 / x \\
-x & 1
\end{array}\right)
$$

$$
A^{-1}=-B, \quad B^{-1}=-A, \quad 1+A^{-1}-B^{-1}=1+A^{-1}+A=0, \quad A-B=-1 .
$$

Since $\alpha \beta=0$ our product is

Since

$$
\left(\begin{array}{ccc}
1 & 0 & c-\alpha A^{-1} \beta \\
0 & 1 & 0 \\
0 & 0 & 1
\end{array}\right) .
$$

$$
(0 a)\left(\begin{array}{cc}
0 & -1 / x \\
x & -1
\end{array}\right)\left(\begin{array}{l}
b \\
0
\end{array}\right)=a b x
$$

it suffices to choose $x \neq c / a b$ and this can be done if $K \neq F_{2}$. Thus $R$ is a center and the proof of the lemma is complete.

Lemma 6. Suppose $G$ has no normal unipotent subgroups save 1 and that $G$ is transitive on $\mathfrak{Q}$, then $G$ is transitive on $\mathbb{C}$.

Proof. Choose a center, $P$, with $a(P)$ maximal. By Lemma $2, Q$ has one orbit of centers containing $P$ and all centers off $a(P)$. If there is another orbit, its members all lie on $a(P)$ and consequently lie on all axes. If $Q$ is a member of this orbit, $Q$ is fixed by $G$ and

$$
\langle X(Q, K)| K \text { an axis for } Q\rangle
$$

is a normal unipotent subgroup not 1 .

Lemma 7. Suppose $K \neq F_{2}$ and $G$ contains no normal unipotent subgroups save 1 . Suppose further that $G$ is transitive on $(5$. Then $V=C \oplus A$ where $C$ and $A$ are stable for $G ; G \mid A=1$, and $Q \mid C=S L(C)$ or $S p(C)$.

Proof. Let $C$ be the sum of the members of $C$ and let $A$ be the intersection of the members of $\mathfrak{A}$. These spaces admit $G$. Suppose $P \subseteq A \cap C$. By Lemma $5, P \in \mathfrak{C}$. But $P \subseteq A$ implies that $P$ is fixed by $G$ and this contradicts the assumption that $G$ has only 1 as a normal unipotent subgroup. Dually $C+A=V$. Certainly $G \mid A=1$. Let $H_{1}$ be a hyperplane of $C$, so $H_{1}+A=H$ is a hyperplane of $V$ and $H \cap C=H_{1}$. Again by the dual of Lemma $5, H$ is an axis for $G$. Suppose $X(P, H) \subseteq G$; then 
$X(P, H) \mid C$ is a subgroup of root type in $S L(C)$ having $P$ as center, $H_{1}$ as axis. Thus every hyperplane of $C$ is an axis for $G \mid C$ and by the dual of Lemma $6 G \mid C$ is transitive on the hyperplanes of $C$ since $G$ is transitive on $C$. Similarly each point of $C$ is a center for $G \mid C$ and $G \mid C$ is transitive on these centers. The conclusion now follows from Lemma 3.

We now can give a proof of the theorem. For this it suffices to suppose $V$ indecomposable. Choose an orbit of centers, $\mathfrak{D}_{1}$, such that $P \in \mathfrak{D}_{1}$ implies $a(P)$ minimal. Set

$$
\begin{aligned}
& G_{1}=\left\langle X(P, H) \mid P \in \mathfrak{D}_{1}\right\rangle, \\
& G_{1}^{*}=\left\langle X(Q, K) \mid Q \notin \mathfrak{D}_{1}\right\rangle .
\end{aligned}
$$

These are normal subgroups of $G$ and by Lemma 2 if $P \in \mathfrak{D}_{1}$ then $G_{1}^{*}$ fixes $P$ and $G_{1}^{*} \mid P=1$. Thus $G_{1} \cap G_{1}^{*}$ is a normal unipotent subgroup so $G_{1} \cap G_{1}^{*}=1$. Since $G=G_{1} \times G_{1}^{*}, G_{1}$ has no normal unipotent subgroups. Also $G_{1}$ is transitive on its set of centers $-\mathfrak{D}_{1}$. If $C_{1}$ is the sum of the centers of $G_{1}$ and $A_{1}$ is the intersection of the axes of $G_{1}$, then from Lemma $7 V=C_{1} \oplus A_{1}$. But $C_{1}$ and $A_{1}$ admit $G_{1}^{*}$ so $A_{1}=0, G_{1}^{*}=1$ and $G=S L(V)$ or $S p(V)$.

Added in proof: Since this paper was submitted for publication, the following paper, containing different but related results obtained by the same basic strategy, has appeared:

F. C. PIPER, On elations of finito projective spaces of odd order. J. London Math. Soc. 41, $641-648(1966)$.

\section{Eingegangen am 27.6. 1966}

Anschrift des Autors:

Jack McLaughlin

Department of Mathematies

The University of Michigan

Ann Arbor (Mich.), USA 